\title{
MÉLAnie GuEnAis
}

\section{Singularité des produits de Anzai associés aux fonctions caractéristiques d'un intervalle}

\author{
Bulletin de la S. M. F., tome 127, no 1 (1999), p. 71-93 \\ <http://www.numdam.org/item?id=BSMF_1999_127_1_71_0>
}

(C) Bulletin de la S. M. F., 1999, tous droits réservés.

L'accès aux archives de la revue «Bulletin de la S. M. F. » (http: //smf.emath.fr/Publications/Bulletin/Presentation.html) implique l'accord avec les conditions générales d'utilisation (http://www.numdam.org/ conditions). Toute utilisation commerciale ou impression systématique est constitutive d'une infraction pénale. Toute copie ou impression de ce fichier doit contenir la présente mention de copyright.

\section{Numdam}




\title{
SINGULARITÉ DES PRODUITS DE ANZAI ASSOCIÉS AUX FONCTIONS CARACTÉRISTIQUES D'UN INTERVALLE
}

\author{
PAR MÉLANIE GUENAIS
}

\begin{abstract}
RÉSUMÉ. - On étudie les extensions à deux points au-dessus d'une rotation irrationnelle définies sur $\mathbb{T} \times \mathbb{Z} / 2 \mathbb{Z}$ par $T_{\beta}(x, y)=\left(x+\alpha \bmod 1, y+\mathbf{1}_{\{0, \beta \mid}(x) \bmod 2\right)$. Ces transformations introduites par A. Katok et A. Stepin admettent un spectre simple lorsqu'elles sont ergodiques, et la question de la nature du type spectral se pose donc naturellement. On établit alors que ces transformations admettent toujours un spectre purement singulier, quels que soient $\alpha \notin \mathbb{Q}$ et $\beta \in] 0,1[$.

AbStraCt. - SpeCtral SINGUlarity OF ANZAi SKEW PRODUCTS ASSOCIATED WITH STEP FUNCTIONS. - Two-point extensions over irrational rotations given on $\mathbb{T} \times \mathbb{Z} / 2 \mathbb{Z}$ by $T_{\beta}(x, y)=\left(x+\alpha \bmod 1, y+\mathbf{1}_{[0, \beta[}(x) \bmod 2\right)$, where $\left.\beta \in\right] 0,1[$ and $\alpha$ is irrational have been extensively studied from the spectral point of view, starting with a well known paper by A. Katok and A. Stepin. In particular, they allways have simple spectrum as soon as they are ergodic, and the question of the existence of such a transformation whose spectral type admits an absolutely continuous part arises naturally. We give here a negative answer to this question, by showing for every $\alpha$ and $\beta \in] 0,1[$ the spectral singularity of these 2 -point extensions.
\end{abstract}

\section{Introduction}

Soient $\alpha \notin \mathbb{Q}$ et $T$ la rotation ergodique sur le tore muni de sa mesure de Lebesgue $(\mathbb{T}, \lambda)$ définie, pour $x \in \mathbb{T}$, par

$$
T x=x+\alpha \quad(\bmod 1) .
$$

Soit $\beta \in] 0,1[$; on étudie la nature du type spectral des produits croisés $T_{\beta}$ définis sur $\mathbb{T} \times \mathbb{Z} / 2 \mathbb{Z}$ par

$$
T_{\beta}(x, y)=\left(T x, y+\mathbf{1}_{[0, \beta[}(y)\right) .
$$

(*) Texte reçu le 22 janvier 1998, révisé le 4 avril 1998, accepté le 24 juin 1998.

M. GuenaIS, Laboratoire de Topologie et Dynamique, UMR D1169 du CNRS, Université Paris-Sud, 91405 Orsay CEDEX. E-mail : guenais@topo.math.u-psud.fr.

Classification AMS : 28D 05, $11 \mathrm{~K} 50$.

Mots clés : théorie ergodique, théorie spectrale, singularité spectrale, produits gauches, rotations, fraction continue.

BULLETIN DE LA SOCIÉTÉ MATHÉMATIQUE DE FRANCE

0037-9484/1999/71/\$5.00

(C) Société mathématique de France 
On peut comparer ces transformations à une autre classe d'extensions très étudiée en théorie ergodique, celle des transformations de $\mathbb{T} \times \mathbb{T}$ définies par $T_{\beta}^{\prime}(x, y)=(T x, y+\beta x)$. Celles-ci jouent un rôle important dans l'étude spectrale des produits de Anzai, et leur singularité spectrale a été établie très récemment grâce à des méthodes de théorie des nombres par A. Iwanik, M. Lemanczyk et C. Mauduit dans [6]. Le but de cet article est d'établir la singularité spectrale des transformations $T_{\beta}$.

Ces produits gauches ont été introduits par A. Katok et A. Stepin en 1966 dans [8] (ou [9]) afin d'exhiber, sous des conditions d'approximations sur $\alpha$ et $\beta$, des exemples de transformations dont le type spectral $\sigma$ vérifie $\sigma * \sigma \nless \sigma$ : il s'agit des premières constructions de systèmes dynamiques mesurables donnant des contre-exemples à la «loi de groupe ».

En 1966, V. Oseledets montre dans [12] l'existence d'une composante spectrale continue pour $T_{1 / 2}$. En fait, la question de l'ergodicité de $T_{\beta}$, ou de l'existence d'une composante spectrale continue est étroitement lié à des problèmes de distribution uniforme des points $(n \alpha)_{n \in \mathbb{Z}}$ dans un intervalle de $\mathbb{T}$ : il s'agit de la première motivation de $W$. Veech dans [16] en 1969. L'auteur donne dans cet article, pour un irrationnel $\alpha$ fixé, un encadrement des $\beta$ pour lesquels $T_{\beta}$ n'est pas ergodique, ou admet un type spectral discret. Lorsque $\alpha$ admet des quotients partiels bornés, le spectre de $T_{\beta}$ est discret si et seulement s'il n'est pas ergodique, c'est-àdire dans ce cas que $\beta \in \alpha \mathbb{Z}$. Ces résultats seront redémontrés grâce à des arguments plus simples par K. Merrill en 1984 dans [11].

On peut citer une autre série de résultats concernant ces transformations, obtenus à l'aide des méthodes d'approximation cyclique développées par A. Katok et A. Stepin. On trouve d'abord dans [8] une majoration de la multiplicité spectrale de $T_{\beta}$ par 2 , lorsque $\alpha$ et $\beta$ sont rationnellement indépendants. En 1976, R.G. Goodson établit dans [1] la simplicité spectrale de $T_{\beta}$, sous certaines conditions d'approximation cyclique : il en déduit dans ce cas l'ergodicité de $T_{\beta}$. Cette méthode est reprise par G. Riley en 1979 dans [15], pour l'étude de la singularité spectrale et la simplicité spectrale de ces transformations. L'auteur obtient ainsi pour tout irrationnel $\alpha$ des résultats presque sûrs en $\beta$, qui généralisent le résultat obtenu dans [8] par A. Katok et A. Stepin.

L'étude spectrale de $T_{\beta}$ consiste en l'étude, sur $L^{2}(\mathbb{T} \times \mathbb{Z} / 2 \mathbb{Z})$, de son opérateur associé

$$
U_{T_{\beta}} f=f \circ T_{\beta} .
$$

Ici, $L^{2}(\mathbb{T} \times \mathbb{Z} / 2 \mathbb{Z})=L_{0} \oplus L_{1}$, où

$$
\begin{aligned}
& L_{0}=\left\{f \otimes 1, f \in L^{2}(X)\right\}, \\
& L_{1}=\left\{f \otimes \operatorname{Id}_{\{-1,1\}}, f \in L^{2}(X)\right\}
\end{aligned}
$$

TOME $127-1999-\mathrm{N}^{\circ} 1$ 
sont deux sous-espaces isomorphes à $L^{2}(\mathbb{T})$, fermés et invariants sous $U_{T_{\beta}}$. Dans ces conditions, l'étude spectrale de $T_{\beta}$ se ramène à l'étude spectrale des restrictions de $U_{T_{\beta}}$ à $L_{0}$ et $L_{1}$ qui sont unitairement équivalentes aux opérateurs $U$ et $V$ définis sur $L^{2}(\mathbb{T})$ par $U f=f \circ T$ et

$$
V f=\phi \cdot f \circ T, \quad \text { où } \quad \phi=\left\{\begin{aligned}
1 & \text { si } x \in[0, \beta[, \\
-1 & \text { sinon. }
\end{aligned}\right.
$$

Par conséquent la multiplicité spectrale de $T_{\beta}$ est majorée par la somme des multiplicités spectrales de $U$ et de $V$. Comme $U$ est l'opérateur d'une rotation ergodique, son spectre est simple et discret. De plus, la simplicité spectrale de $V$ est connue : c'est par exemple une conséquence du théorème 5 de [2], dans le cas d'un cocycle avec deux discontinuités. Finalement, la multiplicité de $T_{\beta}$ vaut 1 dès que $V$ n'admet pas de valeurs propres commune avec $U$ : cette condition de simplicité spectrale est alors équivalente à l'ergodicité de $T_{\beta}(c f .[13])$. On montre ici le résultat général suivant.

ThÉORÈme 1.1. - Soit $T$ une rotation irrationnelle sur le tore, et $\beta \in] 0,1\left[\right.$. Alors le produit croisé $T_{\beta}$ a un spectre purement singulier, simple dès qu'il est ergodique. Plus précisément le spectre de l'opérateur $V$ associé est simple, purement singulier.

Remarque. - Dans [3], H. Helson établit la pureté spectrale de $V$ : c'est-à-dire que son type spectral est soit discret, soit continu et purement singulier, soit équivalent à la mesure de Lebesgue. D'après [4] ou [13], on sait qu'il est en fait ergodique par rapport au sous-groupe des valeurs propres de $T$. Dans ce cas, grâce au théorème 1.1, la loi de pureté telle qu'elle est énoncé dans [5] par exemple montre que lorsque le type spectral de $V$ qu'on notera $\sigma$ est continu, il est étranger à son carré de convolution. Toutes ces transformations donnent ainsi des contre-exemples à la loi de groupe.

En fait, la loi de pureté permet d'affirmer qu'une mesure ergodique vérifie l'une des trois conditions suivantes (cf. [5] par exemple) :

- c'est une mesure discrète;

- l'une de ses puissances de convolution est absolument continue;

- ses puissances de convolution sont deux à deux étrangères.

Comme on montrera en fait que $\varlimsup|\widehat{\lim }| \widehat{\sigma}(n) \mid>0$ (voir la prop. 1.1), on pourra alors affirmer que lorsque $\sigma$ est une mesure continue, ses puissances de convolution sont deux à deux étrangères.

Pour prouver le théorème 1.1, il suffit, en raison de la pureté de $\sigma$, de montrer que la mesure spectrale $\sigma_{1}$ associée à la fonction constante égale 
à 1 n'est pas absolument continue. On va établir plus précisément que $\widehat{\sigma}_{1}(n)$ ne tend pas vers 0 le long d'une suite rigide, c'est-à-dire sur une suite telle que $\|n \alpha\| \rightarrow 0$ (le symbole $\|\cdot\|$ désigne la distance à l'entier le plus proche) : il s'agit là d'une propriété de $\alpha$-mélange pour l'opérateur $V$, qui montre effectivement que $\overline{\lim }|\widehat{\sigma}(n)|>0$ (cf. [7] par exemple).

Soit

$$
\phi^{(n)}(x)= \begin{cases}\phi(x) \phi(T x) \cdots \phi\left(T^{n-1} x\right) & \text { quand } n>0 \\ 1 & \text { si } n=0 \\ \phi^{(-n)}\left(T^{n} x\right) & \text { si } n<0\end{cases}
$$

On a pour tout $n$ l'expression

$$
V^{n} f=\phi^{(n)} f \circ T^{n}
$$

d'où

$$
\widehat{\sigma}_{1}(n)=\int_{\mathbb{T}} \phi^{(n)}(x) \mathrm{d} x=2 \lambda\left(\left\{\phi^{(n)}=1\right\}\right)-1 .
$$

Par conséquent, il suffit de prouver que $\lambda\left(\left\{\phi^{(n)}=1\right\}\right)$ ne tend pas vers $\frac{1}{2}$.

Notons $\left(a_{n}\right)$ la suite des quotients partiels de la fraction continue de $\alpha$, et $\left(q_{n}\right)$ les dénominateurs des réduites associées; le théorème 1.1 est une conséquence du résultat qui suit, vrai pour tout $\beta \in] 0,1[$.

Proposition 1.1. - Il existe un ensemble infini d'entiers $\Lambda$, une suite $\left(\varepsilon_{n}\right)$ de $\{-1,1\}$ et une suite d'entiers $\left(h_{n}\right)$ avec $h_{n} \in\left[q_{n-1}, q_{n+2}\right]$ telles que

$$
\varlimsup_{n \in \Lambda} \lambda\left(\left\{\phi^{\left(h_{n}\right)}=\varepsilon_{n}\right\}\right)<\frac{1}{2} .
$$

La preuve de cette proposition repose sur l'évaluation de $\phi^{\left(h_{n}\right)}$ sur $\mathbb{T}$ lorsque $h_{n} \in\left\{q_{n-1}, q_{n}, q_{n}+q_{n-1}, 2 q_{n}, q_{n+1}, q_{n+2}\right\}$. Comme $\left\|q_{n} \alpha\right\| \rightarrow 0$, les suites $\left(h_{n}\right)$ forment également des suites rigides. On utilise pour cette évaluation, une suite de partitions de $\mathbb{T}$ constituées de deux tours d'intervalles de la rotation. Celles-ci proviennent de la décomposition en fraction continue de $\alpha$ et peuvent être définies par la partition de $\mathbb{T}$ formée par les subdivisions $(\{j \alpha\})_{0 \leq-j<q_{n}+q_{n-1}}$. Ces subdivisions divisent $\mathbb{T}$ en deux tours d'invervalles $\left(T^{-j}\left[0, \alpha_{n-1}[)_{0 \leq j<q_{n}}\right.\right.$ et $\left(T^{-j}\left[-\alpha_{n}, 0[)_{0 \leq j<q_{n-1}}\right.\right.$ (avec $\left.\alpha_{n}=\left\|\alpha q_{n}\right\|\right)$.

Plus précisément, on donne une représentation de ces tours dans $\mathbb{T} \times \mathbb{Z}$ qui permet de mettre en évidence une induction analogue à l'induction de G. Rauzy [14] pour les échanges d'intervalles. On peut alors quantifier facilement la répartition de $\phi^{\left(q_{n}\right)}$ sur $\mathbb{T}$, en fonction de la position relative de 0 et $\beta$.

TOME $127-1999-\mathrm{N}^{\circ} 1$ 
La dernière partie, assez technique, consiste à déterminer, suivant le comportement de la suite des quotients partiels et de $\beta$, les suites $\left(h_{n}\right)$ donnant la majoration voulue.

Je tiens enfin à remercier F. Parreau, pour les conseils utiles qu'il m'a apportés au cours de la réalisation de cet article.

\section{Rappels. Cas des grands quotients partiels}

\subsection{Quelques résultats utiles sur les fractions continues.}

On rappelle d'abord les principales relations liées à la suite des quotients partiels de $\alpha$. On pourra retrouver ces résultats classiques dans [10].

Soient $\alpha_{-1}=1$ et $\alpha_{0}=\alpha$; on définit pour tout $n \geq 0$,

$$
a_{n+1}=\left[\frac{\alpha_{n-1}}{\alpha_{n}}\right] \quad \text { et } \quad \alpha_{n+1}=\alpha_{n-1}-a_{n+1} \alpha_{n} .
$$

Alors la fraction continue $\left[0, a_{1}, \ldots, a_{n}, \ldots\right]$ représente la décomposition en fraction continue de $\alpha$.

Soit $\left(p_{n} / q_{n}\right)$ la suite de réduites associées; elles peuvent être définies par les relations de récurrence suivantes. Notons

$$
q_{-1}=p_{0}=0 \quad \text { et } \quad p_{-1}=q_{0}=1 ;
$$

pour tout $n \geq 0$, on a

$$
p_{n+1}=a_{n+1} p_{n}+p_{n-1} \quad \text { et } \quad q_{n+1}=a_{n+1} q_{n}+q_{n-1} .
$$

On vérifie que

$$
\alpha_{n}=(-1)^{n}\left(q_{n} \alpha-p_{n}\right)=\left\|q_{n} \alpha\right\| .
$$

Comme $\alpha_{n}$ est décroissante et de limite nulle, la suite des réduites converge bien vers $\alpha$. On en déduit les résultats suivants :

$$
\begin{gathered}
a_{n+1} \alpha_{n}<\alpha_{n-1}<\left(1+a_{n+1}\right) \alpha_{n}, \\
a_{n+1} q_{n}<q_{n+1}<\left(1+a_{n+1}\right) q_{n}, \\
\alpha_{n+1} q_{n}+q_{n+1} \alpha_{n}=1 .
\end{gathered}
$$

Dans le cas où la suite $\left(a_{n}\right)$ est bornée, on a pour tous $(j, k)$ fixés dans $\mathbb{Z}$, les inégalités

$$
\begin{aligned}
& \varliminf_{n \rightarrow \infty} \alpha_{n+j} q_{n+k}>0, \\
& \varlimsup_{n \rightarrow \infty} \alpha_{n+1} q_{n}<\frac{1}{2} .
\end{aligned}
$$


En effet, si $j<k$, on a $\alpha_{n+j} \geq \alpha_{n+k-1}$ d'où

$$
\alpha_{n+j} q_{n+k} \geq \alpha_{n+k-1} q_{n+k} \geq \frac{1}{2}
$$

Si $j \geq k$, soit $M$ un majorant de $\left(a_{n}\right)$; on a

$$
\alpha_{n+j-1} \leq(M+1) \alpha_{n+j}
$$

d'où on déduit que

$$
\alpha_{n+j} q_{n+k} \geq \alpha_{n+k-1} q_{n+k}(M+1)^{-(j-k+1)} \geq \frac{1}{2}(M+1)^{-(j-k+1)} .
$$

Ceci donne l'inégalité (1). La seconde relation en est une conséquence directe, en écrivant simplement

$$
1=\alpha_{n+1} q_{n}+\alpha_{n} q_{n+1} \geq 2 \alpha_{n+1} q_{n}+\alpha_{n+2} q_{n+1}
$$

Enfin dans les cas où la suite $\left(a_{n}\right)$ est constante, on aura besoin des équivalences suivantes :

LEMME 2.1. - Si $\lim _{n \rightarrow \infty} a_{n}=1$, on $a \lim _{n \rightarrow \infty} \alpha_{n} q_{n}=1 / \sqrt{5}$.

Preuve. - Pour tout $n$ assez grand, on a

$$
q_{n+1}=q_{n}+q_{n-1} \quad \text { et } \quad \alpha_{n+1}=\alpha_{n-1}-\alpha_{n} .
$$

Comme en plus $q_{n} \rightarrow+\infty$ et $\alpha_{n} \rightarrow 0$, on obtient donc $q_{n} \sim Q \theta^{n}$ et $\alpha_{n} \sim \mathcal{A}(1 / \theta)^{n}$ avec $\theta=\frac{1}{2}(\sqrt{5}+1)$. D'autre part,

$$
\alpha_{n} q_{n-1}+\alpha_{n-1} q_{n}=1 \text { pour tout } n,
$$

ce qui montre que $Q \mathcal{A}=(\theta+1 / \theta)^{-1}=1 / \sqrt{5}$, d'où le résultat.

De la même façon on obtient :

Lemme 2.2. - Si $\lim _{n \rightarrow \infty} a_{n}=3$, on a $\alpha_{n} \sim \mathcal{A} \theta^{-n}$ et $q_{n} \sim Q \theta^{n}$, où $\theta=\frac{1}{2}(\sqrt{13}+3)$ et $Q \mathcal{A}=1 / \sqrt{13}$.

\subsection{Démonstration de la proposition dans certains cas.}

Si les quotients partiels de $\alpha$ sont assez grands, on peut démontrer facilement la proposition 1.1.

TOME $127-1999-\mathrm{N}^{\circ} 1$ 
Lemme 2.3. - La proposition 1.1 est vraie pour tout $\beta \in] 0,1[$ lorsque la suite des quotients partiels de $\alpha,\left(a_{n}\right)$ vérifie l'une des conditions suivantes :

- $\varlimsup_{n \rightarrow+\infty} a_{n} \geq 4$;

- il existe une infinité d'entiers $n$ tels que $a_{n}=1, a_{n+1}=3$ et $a_{n+2}=1$.

Preuve. - On considère la répartition du signe de $\phi^{\left(2 q_{n}\right)}$ dans $\mathbb{T}$. On peut se restreindre au cas où $n$ est pair; on a alors

$$
T^{q_{n}} x=x+\alpha_{n}
$$

Alors $\phi^{\left(q_{n}\right)}=\prod_{0}^{q_{n}-1} \phi \circ T^{j}$, et $\phi^{\left(q_{n}\right)}$ admet donc $2 q_{n}$ discontinuités,

$$
\left\{T^{-j} 0, T^{-j} \beta\right\}_{0 \leq j<q_{n}} .
$$

Comme $\phi^{\left(2 q_{n}\right)}=\phi^{\left(q_{n}\right)} \phi^{\left(q_{n}\right)} \circ T^{q_{n}}$, elle possède toutes les discontinuités de $\phi^{\left(q_{n}\right)}$ ainsi que leurs translatées par $\alpha_{n}$. La fonction $\phi^{\left(2 q_{n}\right)}$ a alors un signe constant sur le complémentaire $I$ de

$$
\bigcup_{0 \leq j<q_{n}}\left(T^{-j}\left[-\alpha_{n}, 0\right] \cup T^{-j}\left[\beta-\alpha_{n}, \beta\right]\right)
$$

En effet si $x \in I$, l'intervalle $\left[x, x+\alpha_{n}\right.$ [ ne contient aucune des discontinuités de $\phi^{\left(q_{n}\right)}$, d'où $\phi^{\left(q_{n}\right)}(x)=\phi^{\left(q_{n}\right)}\left(x+\alpha_{n}\right)$ soit $\phi^{\left(2 q_{n}\right)}(x)=$ $\phi^{\left(q_{n}\right)}(x) \phi^{\left(q_{n}\right)}\left(x+\alpha_{n}\right)=1$. On obtient donc :

$$
\lambda\left(\phi^{\left(2 q_{n}\right)}=-1\right) \leq \lambda(\mathbb{T} \backslash I) \leq 2 \lambda\left(\bigcup_{0 \leq j<q_{n}} T^{-j}\left[-\alpha_{n}, 0\right]\right) \leq 2 \alpha_{n} q_{n} .
$$

Si $\varlimsup a_{n}>4$, il existe un ensemble infini $\Lambda$ d'entiers $n$ tels que $a_{n+1}>4$ et on a alors pour tout $n \in \Lambda$

$$
\alpha_{n} q_{n} \leq \frac{\alpha_{n-1} q_{n}}{a_{n+1}} \leq \frac{1}{5}
$$

Si $\varlimsup a_{n}=4$, l'ensemble $\Lambda$ des $n$ tels que $a_{n+1}=4$ est infini. Comme dans ce cas la suite $\left(a_{n}\right)$ est bornée, on a $\varlimsup$ lim $\alpha_{n} q_{n-1}<1$, d'où

$$
\varlimsup_{n \in \Lambda} \alpha_{n} q_{n} \leq \varlimsup_{n \in \Lambda} \frac{\alpha_{n-1} q_{n}}{a_{n+1}}<\frac{1}{4}
$$


Enfin, si $\varlimsup a_{n} \leq 3$, et si $n$ vérifie la seconde condition du lemme, on a

$$
\alpha_{n-1}=3 \alpha_{n}+\alpha_{n+1}, \quad \alpha_{n}=\alpha_{n+1}+\alpha_{n+2}, \quad q_{n}=q_{n-1}+q_{n-2},
$$

d'où :

$$
\begin{aligned}
1 & =\alpha_{n-1} q_{n}+\alpha_{n} q_{n-1} \\
& =3 \alpha_{n} q_{n}+\alpha_{n+1} q_{n}+\alpha_{n}\left(q_{n}-q_{n-2}\right) \\
& =4 \alpha_{n} q_{n}+\alpha_{n+1} q_{n}-q_{n-2}\left(\alpha_{n+1}+\alpha_{n+2}\right) \\
& =4 \alpha_{n} q_{n}+\alpha_{n+1} q_{n-1}-\alpha_{n+2} q_{n-2} \\
& \geq 4 \alpha_{n} q_{n}+\alpha_{n+2} q_{n-3} .
\end{aligned}
$$

Par conséquent si $\left(a_{n}\right)$ vérifie la seconde condition du lemme, il existe un ensemble infini $\Lambda$ d'entiers $n$ tels que

$$
\alpha_{n} q_{n} \leq \frac{1}{4}\left(1-\alpha_{n+2} q_{n-3}\right) .
$$

Comme $\left(a_{n}\right)$ est bornée dans ce cas, on a $\varliminf_{n \rightarrow \infty} \alpha_{n+2} q_{n-3}>0$, d'où $\varlimsup_{n \in \Lambda} 2 \alpha_{n} q_{n}<\frac{1}{2}$. On a finalement trouvé un ensemble infini d'entiers $\Lambda$, tel que

$$
\varlimsup_{n \in \Lambda} \lambda\left(\phi^{\left(2 q_{n}\right)}=-1\right)<\frac{1}{2}
$$

ce qui montre la proposition 1.1 pour tout $\beta$.

\section{Représentation sur $\mathbb{T} \times \mathbb{Z}$}

D'après le lemme 2.3 , dans toute la suite on supposera que

$$
\varlimsup_{n \rightarrow \infty} a_{n} \leq 3 \text {. }
$$

\subsection{Domaines fondamentaux associés aux tours de la rotation.}

On a vu que la décomposition en fraction continue d'un nombre irrationnel $\alpha$ fait apparaître une suite de partitions de $\mathbb{T}$ constituées de deux tours d'intervalles. Pour visualiser facilement l'induction sur ces tours, on utilise une représentation dans $\mathbb{T} \times \mathbb{Z}$ qui est définie de la façon suivante.

Soit $\pi$ la projection de $\mathbb{T} \times \mathbb{Z}$ dans $\mathbb{T}$ définie par

$$
\pi(x, k)=T^{k} x \text {. }
$$

Alors $\mathbb{T}$ est isomorphe au quotient de $\mathbb{T} \times \mathbb{Z}$ par $\pi$, et la rotation $T$ correspond à l'une ou l'autre des translations de $\mathbb{T} \times \mathbb{Z}$ définies par

$$
T_{v}(x, k)=(x, k+1) \quad \text { et } \quad T_{h}(x, k)=(T x, k),
$$

c'est-à-dire que $\pi \circ T_{v}=\pi \circ T_{h}=T \circ \pi$. On représente maintenant $\mathbb{T}$ dans $\mathbb{T} \times \mathbb{Z}$ par un domaine fondamental, c'est-à-dire par une partie mesurable $\mathcal{D}$

TOME $127-1999-\mathrm{N}^{\circ} 1$ 
de $\mathbb{T} \times \mathbb{Z}$ sur laquelle la restriction de $\pi$ est une bijection. La famille $\left\{T_{v}^{n} \circ T_{h}^{-n} \mathcal{D}, n \in \mathbb{Z}\right\}$ forme alors un pavage de $\mathbb{T} \times \mathbb{Z}$.

Pour tout $n$, on choisit un domaine fondamental $\mathcal{D}_{n}$ qui représente les deux tours d'intervalles de la rotation définies précédemment. On le définit pour $n$ pair par :

$\mathcal{D}_{n}=\left[-\alpha_{n}, \alpha_{n-1}\left[\times\left\{1, \ldots, q_{n-1}\right\} \cup\left[-\alpha_{n}, \alpha_{n-1}-\alpha_{n}\left[\times\left\{q_{n-1}+1, \ldots, q_{n}\right\}\right.\right.\right.\right.$.

Si $n$ est impair, il est plus commode de changer l'orientation de l'axe des abscisses, et dans ce cas la définition de $\mathcal{D}_{n}$ est la même que pour $n$ pair. $\left(\mathcal{D}_{n}\right)$ définit ainsi une suite de domaines fondamentaux associée à la fraction continue de $\alpha$.

$\grave{A}$ tout $\beta$ de $\mathbb{T}$, on associe son unique représentant $\beta_{n}$ dans $\mathcal{D}_{n}$; ses coordonnées $\left((-1)^{n} x_{n}, k_{n}\right)$ sont données par la décomposition

$$
\beta=k_{n} \alpha+(-1)^{n} x_{n}
$$

où $\left(x_{n}, k_{n}\right) \in \mathcal{D}_{n}$ (exprimées dans le repère «local» lié à la parité de $n$ ). La figure 1 met en évidence le pavage associé à $\mathcal{D}_{n}$ sur $\mathbb{T} \times \mathbb{Z}$.

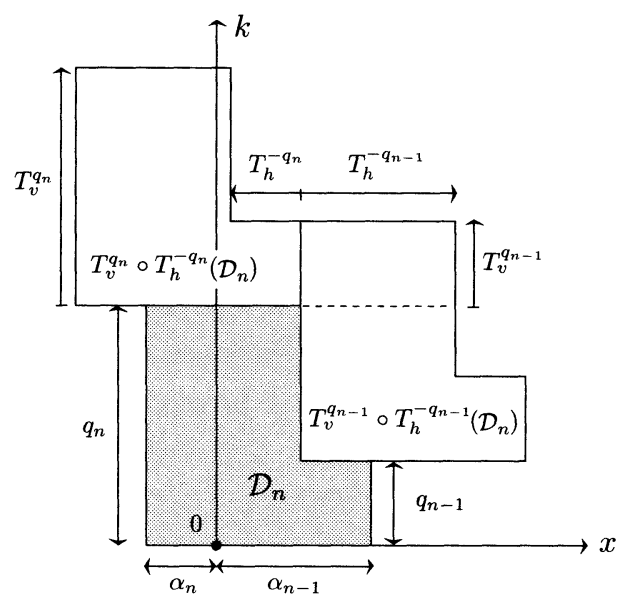

Figure 1

La décomposition de $\mathcal{D}_{n+1}$ sur le pavage associé à $\mathcal{D}_{n}$ donne l'induction entre les domaines fondamentaux. Celle-ci permet d'expliciter graphiquement les récurrences sur la suite des représentants $\beta_{n}$ de $\beta$ dans $\mathcal{D}_{n}$. On retrouve aisément les relations de récurrence attenant à la fraction continue de $\alpha$. 


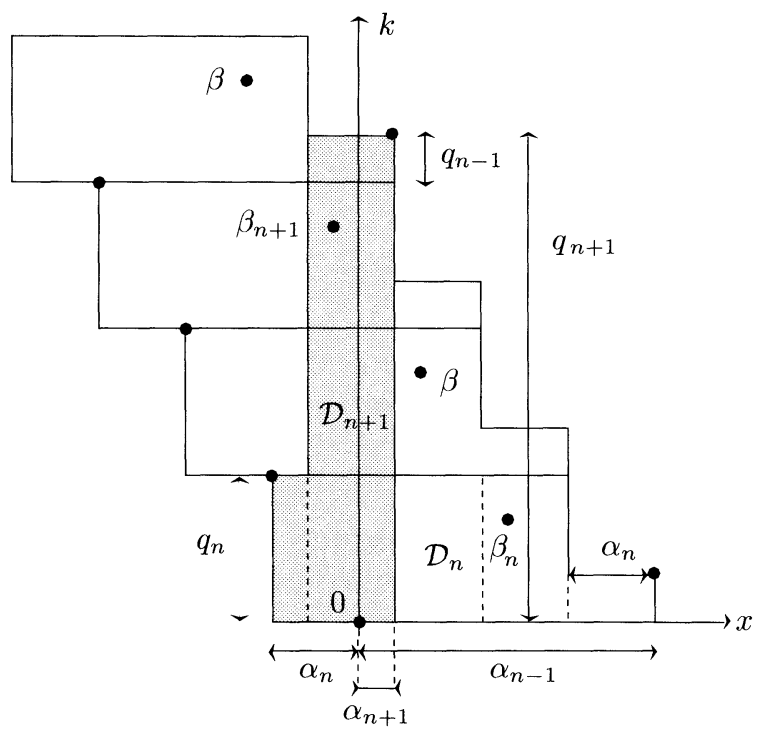

Figure 2

Graphiquement (voir figure 2), si $\beta \in \mathbb{T}$, on notera $\beta$ n'importe lequel de ses représentants dans $\mathbb{T} \times \mathbb{Z}$. De la même façon, une partie de $\mathcal{D}_{n}$ sera identifiée à sa projection sur $\mathbb{T}$, et donc à ses translatés par $T_{v} \circ T_{h}{ }^{-1}$.

\subsection{Répartition de $\phi^{\left(q_{n}\right)}$.}

Dans le cadre de le lemme 2.3, les positions relatives des discontinuités de $\phi^{\left(q_{n}\right)}$ n'interviennent pas. Chacune d'elles est en effet couplée avec sa translatée par $T^{q_{n}}$ qui «annule» le changement de signe de $\phi^{\left(2 q_{n}\right)}$. Lorsque $\varlimsup_{n \rightarrow \infty} a_{n} \leq 3$, l'évaluation précédente n'est pas suffisante et on est donc amené à regarder précisément la répartition des discontinuités de $\phi^{\left(q_{n}\right)}$. Pour cela, on représente dans $\mathcal{D}_{n}$ la distribution du signe de $\phi^{\left(q_{n}\right)}$ en fonction de la position du représentant de $\beta$ dans $\mathcal{D}_{n}$.

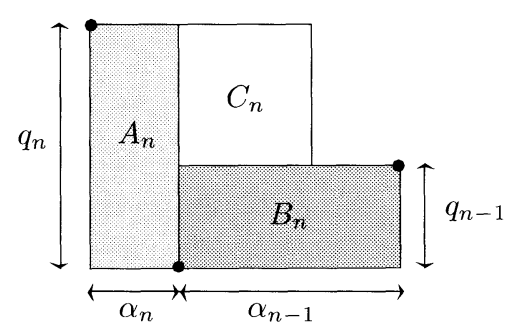

Figure 3

TOME $127-1999-\mathrm{N}^{\circ} 1$ 
Dans le repère d'ordre $n$, on a :

$$
\begin{aligned}
& A_{n}=\left[-\alpha_{n}, 0\right] \times\left\{1, \ldots, q_{n}\right\}, \\
& B_{n}=\left[0, \alpha_{n-1}\right] \times\left\{1, \ldots, q_{n-1}\right\}, \\
& C_{n}=\left[0, \alpha_{n-1}\right] \times\left\{q_{n-1}+1, \ldots, q_{n}\right\} .
\end{aligned}
$$

On vérifie aisément que $A_{n-1}=B_{n}$. Par conséquent, $B_{n} \subset A_{n+1} \cup C_{n+1}$ et $C_{n} \subset A_{n+1} \cup C_{n+1}$.

En fait, on divise $\mathbb{T}$ en trois parties $\left(A_{n}, B_{n}, C_{n}\right)$ dont les représentants dans $\mathcal{D}_{n}$ sont disposés figure 3 . Comme 0 est invariant pour la suite $\left(\mathcal{D}_{n}\right)$, la répartition des signes de $\phi^{\left(q_{n}\right)}$ ne dépend donc que de la position de $\beta_{n}$ dans $\mathcal{D}_{n}$.

Supposons $n$ pair (sinon le raisonnement est le même par symétrie par rapport à l'axe vertical) : on a $T^{q_{n}} x=x+\alpha_{n}(\bmod 1)$. On rappelle que $\phi^{\left(q_{n}\right)}$ possède $2 q_{n}$ discontinuités aux points $\left\{T^{-j} 0, T^{-j} \beta\right\}_{0 \leq j<q_{n}}$. Comme chaque étage de $\mathcal{D}_{n}$ correspond à un intervalle de $\mathbb{T}, \phi^{\left(q_{n}\right)}$ ne change donc de signe sur un étage que s'il contient une discontinuité. Soit maintenant $I$ un segment d'un étage de $\mathcal{D}_{n}$ ne contenant pas de discontinuité. On compare la valeur de $\phi^{\left(q_{n}\right)}$ sur $I$ avec sa valeur sur sur $T_{v} I$. On a l'égalité

$$
\phi^{\left(q_{n}\right)}(T x)=\phi^{\left(q_{n}\right)}(x) \phi\left(T^{q_{n}} x\right) / \phi(x) .
$$

Alors $\phi^{\left(q_{n}\right)}(T x)$ est de même signe que $\phi^{\left(q_{n}\right)}(x)$ si et seulement si $] x, x+\alpha_{n}$ ] ne contient ni 0 ni $\beta$ (c'est vrai dès que $\alpha_{n}<\|\beta\|$ ). Par conséquent, si $I \cap\left(\left[T^{-q_{n}} 0,0\left[\cup\left[T^{-q_{n}} \beta, \beta[)=\emptyset\right.\right.\right.\right.$ on a

$$
\phi^{\left(q_{n}\right)}(I)=\phi^{\left(q_{n}\right)}(T(I)) .
$$

On obtient alors la répartition du signe de $\phi^{\left(q_{n}\right)}$ dans $\mathcal{D}_{n}$, suivant la position de $\beta_{n}$ dans $\mathcal{D}_{n}$. Deux cas se présentent, suivant que $\beta$ appartient à $A_{n}$ ou non.

3.2.1. $\beta$ n'est pas dans $A_{n}$. - Supposons d'abord que $\beta$ ne soit pas dans $A_{n}$, c'est-à-dire que ses coordonnées locales dans $\mathcal{D}_{n}$ vérifient $x_{n}>0$. Dans ce cas, on obtient la répartition du signe de $\phi^{\left(q_{n}\right)}$ dans $\mathcal{D}_{n}$ selon la figure 4. Soit $\varepsilon_{n}=(-1)^{n} \phi^{\left(q_{n}\right)}(0)$ (correspondant au signe de $\phi^{\left(q_{n}\right)}$ dans la partie grisée de la figure 4$)$; il vient

$$
\lambda\left(\phi^{\left(q_{n}\right)}=\varepsilon_{n}\right)=k_{n} \alpha_{n}+q_{n} x_{n}
$$

On constate que lorsque $\beta$ varie dans $\mathbb{T} \backslash A_{n}$, cette quantité varie beaucoup. On sera donc obligé de redécouper cette partie pour obtenir des majorations satisfaisantes. (On peut remarquer que dans ce cas on a aussi $\phi^{\left(q_{n}\right)}(0)=-\phi^{\left(q_{n}\right)}(\beta)$.) 


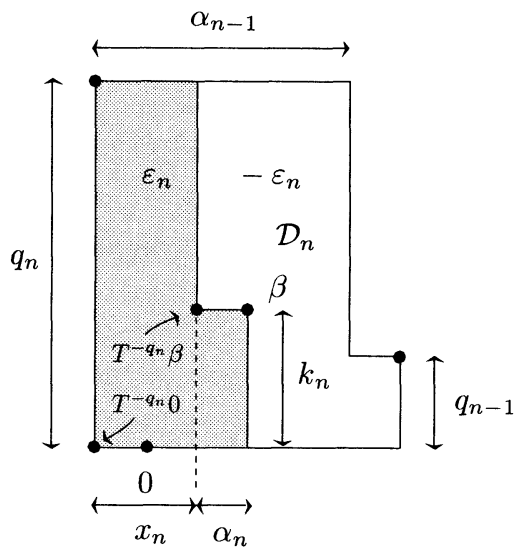

Figure 4

3.2.2. $\beta$ appartient $\grave{a} A_{n}$. $-\mathrm{Si} \beta \in A_{n}$, la répartition est un peu différente de la précédente (figure 5 ). Soit $\left(x_{n}, k_{n}\right)$ les coordonnées locales de $\beta$ dans $\mathcal{D}_{n}$, on a $-\alpha_{n} \leq x_{n}<0$. La valeur de $\phi^{\left(q_{n}\right)}$ dans la partie grise est encore égale à $\varepsilon_{n}$. On obtient alors

$$
\lambda\left(\phi^{\left(q_{n}\right)}=\varepsilon_{n}\right)=-\left(q_{n}-k_{n}\right) x_{n}+k_{n}\left(\alpha_{n}+x_{n}\right) .
$$

Il en résulte, pour tout $\beta$ dans $A_{n}$, la majoration :

$$
\lambda\left(\phi^{\left(q_{n}\right)}=\varepsilon_{n}\right) \leq \alpha_{n} q_{n} .
$$

\section{Résultats intermédiaires}

Lemme 4.1. - S'il existe une infinité d'entiers $n$ tels que $a_{n+1} \geq 2$ et $\beta \in A_{n}$, alors la proposition 1.1 est vraie.

Preuve. - Soit $\Lambda$ l'ensemble infini des entiers vérifiant les hypothèses du lemme. Si $n \in \Lambda$, comme $a_{n+1} \neq 1$, on a $\alpha_{n} q_{n} \leq \frac{1}{2} \alpha_{n-1} q_{n}$, et d'après (1), on obtient $\varlimsup_{n \in \Lambda} \alpha_{n} q_{n} \leq \frac{1}{2} \varlimsup_{n \rightarrow \infty} \alpha_{n-1} q_{n}<\frac{1}{2}$. De plus on a aussi $\beta \in A_{n}$, et la proposition 1.1 résulte simplement de l'inégalité (5).

Lemme 4.2. - S'il existe une infinité d'entiers $n$ tels que $\beta \in C_{n}$ et $a_{n+1}=1$, alors la proposition 1.1 est vraie.

Preuve. - Si $n$ vérifie les conditions du lemme alors $\alpha_{n-1}=\alpha_{n}+\alpha_{n+1}$, d'où la figure 6 . Dans le repère d'ordre $n$, on peut écrire :

$$
\begin{gathered}
C_{n}=\left[0, \alpha_{n+1}\right] \times\left\{q_{n-1}+1, \ldots, q_{n}\right\}, \\
A_{n+1}=\left[0, \alpha_{n+1}\right] \times\left\{1, \ldots, q_{n+1}\right\} .
\end{gathered}
$$

TOME $127-1999-\mathrm{N}^{\circ} 1$ 


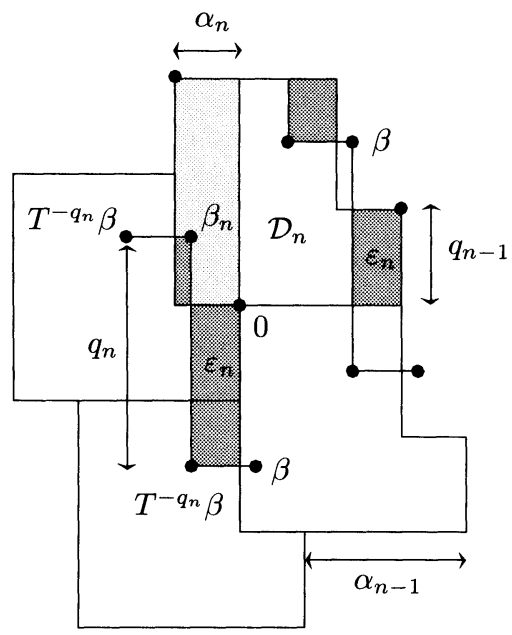

Figure 5

Si $\beta \in C_{n}$, alors $0 \leq x_{n}<\alpha_{n+1}$, et donc $\beta_{n}=\beta_{n+1}$. On en déduit que $\beta \in A_{n+1}$, et l'égalité (4) à l'ordre $n+1$, donne

$$
\lambda\left(\phi^{\left(q_{n+1}\right)}=\varepsilon_{n+1}\right)=x_{n}\left(q_{n+1}-k_{n}\right)+k_{n}\left(\alpha_{n+1}-x_{n}\right) .
$$

La valeur maximale de cette expression lorsque $\left(x_{n}, k_{n}\right) \in C_{n}$ est obtenue sur les bords (il n'y a pas d'extremum local) et donne finalement

$$
\lambda\left(\phi^{\left(q_{n+1}\right)}=\varepsilon_{n+1}\right) \leq \alpha_{n+1} q_{n}
$$

pour tout $\beta \in C_{n}$. Il existe donc un ensemble infini $\Lambda$ d'entiers $n$ vérifiant l'inégalité précédente. D'après le lemme 2.3 , on peut supposer que $\varlimsup$ lim $a_{n} \leq 3$, et l'inégalité (2) donne $\varlimsup_{n \rightarrow \infty} \alpha_{n+1} q_{n}<\frac{1}{2} ;$ on en conclut que

$$
\varlimsup_{n \in \Lambda} \lambda\left(\phi^{\left(q_{n+1}\right)}=\varepsilon_{n+1}\right)<\frac{1}{2}
$$

ce qui montre la proposition 1.1.

LemME 4.3. - Soit $\Lambda$ un ensemble infini d'entiers $n$ tels que $a_{n+1} \neq 1$; alors on $a$ :

$$
\varlimsup_{n \in \Lambda}\left(\alpha_{n} q_{n}+\alpha_{n+1} q_{n-1}\right)<\frac{1}{2} .
$$

Preuve. - Par définition, si $n \in \Lambda$, on a

$$
\alpha_{n-1}=a_{n+1} \alpha_{n}+\alpha_{n+1} \geq 2 \alpha_{n}+\alpha_{n+1} .
$$




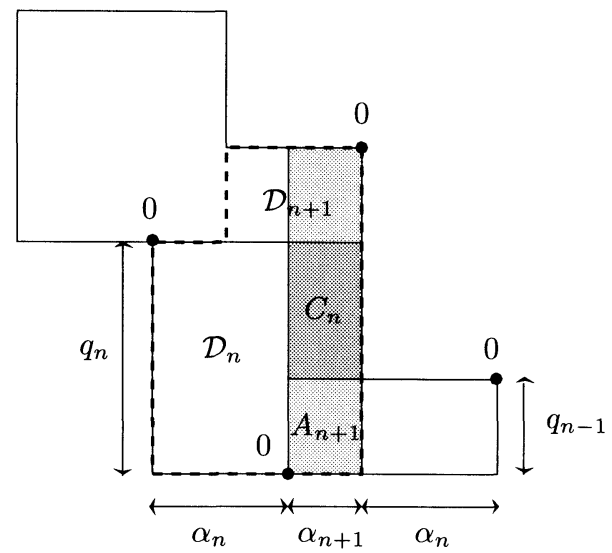

Figure 6

Il vient alors :

$$
\begin{aligned}
1 & =\alpha_{n} q_{n-1}+\alpha_{n-1} q_{n} \\
& \geq 2 \alpha_{n} q_{n}+\alpha_{n+1} q_{n}+\alpha_{n} q_{n-1} \\
& \geq 2\left(\alpha_{n} q_{n}+\alpha_{n+1} q_{n-1}\right)+\alpha_{n+2} q_{n-1} .
\end{aligned}
$$

Comme $\varliminf_{n \rightarrow \infty} \alpha_{n+2} q_{n-1}>0$ d'après (1), on en déduit l'inégalité cherchée.

On choisit maintenant, à partir de la répartition de $\phi^{\left(q_{n}\right)}$, des ensembles infinis d'entiers qui dépendent du comportement asymptotique de la suite des quotients partiels de $\alpha$. Pour démontrer la proposition 1.1 il suffit d'après le lemme 2.3 de distinguer seulement les cas suivants :

(i) il existe $n_{0}$ tel que $a_{n}=1$ pour $n \geq n_{0}$;

(ii) il existe $n_{0}$ tel que $a_{n}=3$ pour $n \geq n_{0}$;

(iii) il existe une infinité de $n$ tels que $a_{n} \neq 1, a_{n+1}=3$ et $a_{n+2}=1$;

(iv) il existe une infinité de $n$ tels que $a_{n+1}=2$, mais on n'a pas (iii).

On définit alors l'ensemble infini $\Lambda$ correspondant, c'est-à-dire qu'on a, suivant les cas :

(i) $\Lambda=\left\{n, a_{n}=1\right\}$;

(ii) $\Lambda=\left\{n, a_{n}=3\right\}$;

(iii) $\Lambda=\left\{n, a_{n} \neq 1, a_{n+1}=3, a_{n+2}=1\right\}$;

(iv) $\Lambda=\left\{n, a_{n+1}=2\right\}$.

TOME $127-1999-\mathrm{N}^{\circ} 1$ 
Empiriquement, pour chaque $n \in \Lambda$ fixé, l'idée est de chercher à l'aide de l'induction sur les tours de la rotation, des entiers voisins de $n$ pour lesquels $\beta$ est «proche» de $A_{n}$.

\section{Démonstration de la proposition lorsque $\varlimsup$ lim $a_{n} \leq 3$}

\section{1. $\beta$ est dans $A_{n}$.}

On suppose que $\Lambda_{A}=\Lambda \cap\left\{n, \beta \in A_{n}\right\}$ est infini. Si $a_{n+1} \neq 1$ pour $n \in \Lambda$, la proposition 1.1 résulte directement du lemme 4.1 : ceci règle tous les cas sauf (i). Dans le cas (i), on a aussi $\varlimsup_{n \rightarrow \infty} \alpha_{n} q_{n}<\frac{1}{2}$ d'après le lemme 2.1, et l'inégalité (5) donne

$$
\varlimsup_{n \in \Lambda_{A}} \lambda\left(\phi^{\left(q_{n}\right)}=\varepsilon_{n}\right)<\frac{1}{2}
$$

ce qui démontre la proposition 1.1 .

\section{2. $\beta$ est dans la partie $B_{n}$.}

On suppose que $\beta \in B_{n}$ pour $n \in \Lambda$ assez grand. Comme $B_{n}=A_{n-1}$, le cas (i) se ramène au paragraphe précédent. Si pour une infinité de $n \in \Lambda$ on a $a_{n} \neq 1$, la proposition 1.1 est encore une conséquence du lemme 4.1 : cette condition est vérifiée pour (ii) et (iii). Dans le cas (iv), elle est encore vraie s'il existe une infinité de $n \in \Lambda$ tels que $a_{n} \neq 1$.

Le dernier cas de la condition (iv) à traiter est donc le suivant : on peut choisir $n_{0}$ tel que pour tout $n \in \Lambda$ plus grand que $n_{0}$, on a $a_{n}=1$ et $a_{n+1}=2$; on va montrer qu'alors, $a_{n+2}=1$.

Comme la condition (iii) n'est pas vérifiée, on peut choisir $n_{0}$ tel que pour tout $n \geq n_{0}$, on n'ait pas $\left(a_{n} \neq 1, a_{n+1}=3, a_{n+2}=1\right)$. Soit $n \in \Lambda$, supérieur à $n_{0}$ : par hypothèse $a_{n+1} \neq 1$ d'où $n+1 \notin \Lambda$, et $a_{n+2}$ vaut donc soit 1 soit 3 . Supposons que $a_{n+2}=3$, on définit alors

$$
k_{n}=\min \left\{k>n+1, a_{k+1} \neq 3\right\} \text {; }
$$

comme $a_{k_{n}}=3, k_{n} \notin \Lambda$ et $a_{k_{n}+1} \neq 2$. On aurait alors un entier $k_{n}-1 \geq n_{0}$ tel que $a_{k_{n}-1} \neq 1\left(\operatorname{car} k_{n}-1>n\right), a_{k_{n}}=3$ et $a_{k_{n}+1}=1$, ce qui est imposssible.

Il reste donc à démontrer la proposition lorsque

$$
a_{n}=1, \quad a_{n+1}=2 \text { et } a_{n+2}=1
$$

pour $n \in \Lambda$. On décompose alors $B_{n}$ sur $A_{n+1}$ et $C_{n+1}$. Comme $a_{n+2}=1$, s'il existe une infinité de $n \in \Lambda$ pour lesquels $\beta \in B_{n} \cap C_{n+1}$, la proposition 1.1 découle du lemme 4.2. Sinon, on a $\beta \in B_{n} \cap A_{n+1}$ pour tout $n \in \Lambda$ assez grand et dans ce cas on distingue trois parties $B_{n}^{1}, B_{n}^{2}$, et $B_{n}^{3}$ dans $A_{n+1} \cap B_{n}$, qu'on peut représenter sur la figure 7 . 


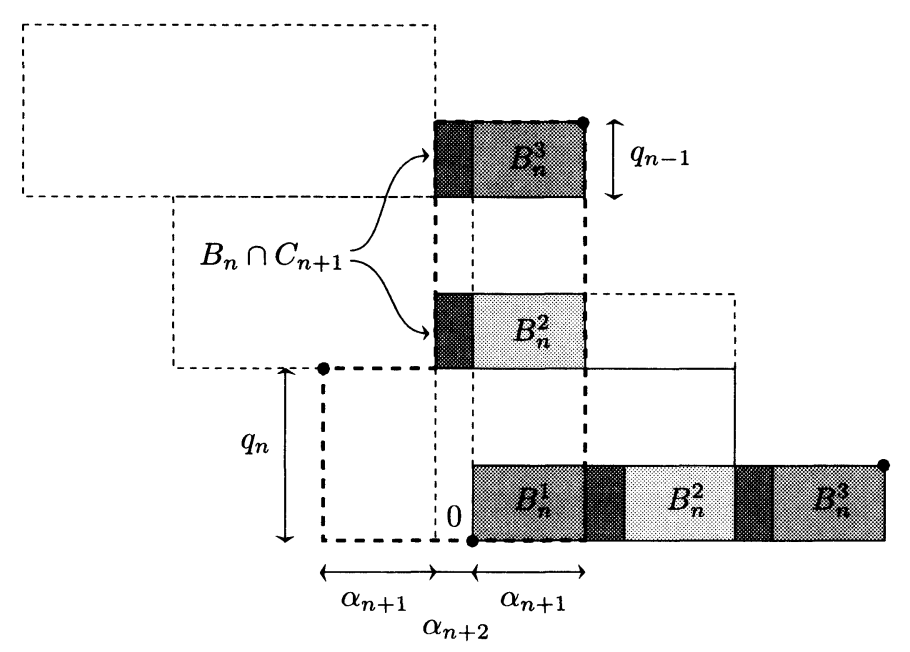

Figure 7

Dans le repère d'ordre $n$, on a :

$$
\begin{aligned}
& B_{n}^{1}=\left[0, \alpha_{n+1}\right] \times\left\{1, \ldots, q_{n-1}\right\} \\
& B_{n}^{2}=\left[0, \alpha_{n+1}\right] \times\left\{q_{n}+1, \ldots, q_{n}+q_{n-1}\right\} \\
& B_{n}^{3}=\left[\alpha_{n-1}-\alpha_{n+1}, \alpha_{n-1}\right] \times\left\{1, \ldots, q_{n-1}\right\} .
\end{aligned}
$$

- Si $\beta \in B_{n}^{1}$, avec l'inégalité (3) à l'ordre $n$, il vient

$$
\begin{aligned}
\lambda\left(\phi^{\left(q_{n}\right)}=\varepsilon_{n}\right) & =k_{n} \alpha_{n}+q_{n} x_{n} \\
& \leq q_{n-1} \alpha_{n}+q_{n} \alpha_{n+1} \\
& \leq q_{n} \alpha_{n}+q_{n-1} \alpha_{n+1} .
\end{aligned}
$$

Comme $a_{n+1} \neq 1$, on est dans le cadre du lemme 4.3 d'où

$$
\varlimsup_{\substack{n \in \Lambda \\ \beta \in B_{n}^{1}}} \lambda\left(\left\{\phi^{\left(q_{n}\right)}=\varepsilon_{n}\right\}\right)<\frac{1}{2} .
$$

- Si $\beta \in B_{n}^{3}$ : alors $0 \in B_{n}^{1}$ dans le domaine fondamental d'origine $\beta$. On obtient donc la même majoration que dans le cas précédent, en intervertissant les rôles de $\beta$ et 0 , ce qui revient à remplacer $\varepsilon_{n}$ par $-\varepsilon_{n}$ dans l'inégalité précédente.

TOME $127-1999-\mathrm{N}^{\circ} 1$ 
- Si $\beta \in B_{n}^{2}$, comme $B_{n}^{2} \subset A_{n+1}$, l'égalité (4) à l'ordre $n+1$ donne pour tout $\beta \in B_{n}^{2}$

$$
\begin{aligned}
\lambda\left(\left\{\phi^{\left(q_{n+1}\right)}=\varepsilon_{n+1}\right\}\right) & =-x_{n+1}\left(q_{n+1}-k_{n+1}\right)+k_{n+1}\left(\alpha_{n+1}+x_{n+1}\right) \\
& \leq\left(q_{n-1}+q_{n}\right) \alpha_{n+1} \\
& \leq q_{n-1} \alpha_{n+1}+q_{n} \alpha_{n},
\end{aligned}
$$

d'où le résultat grâce au lemme 4.3.

\section{3. $\boldsymbol{\beta}$ est dans la partie $\boldsymbol{C}_{\boldsymbol{n}}$.}

On suppose maintenant que $\Lambda_{C}=\left\{n \in \Lambda, \beta \in C_{n}\right\}$ est infini.

5.3.1. Cas (i). - Comme dans ce cas $a_{n+1}=1$, on obtient la proposition 1.1 grâce au lemme 4.2 .

5.3.2. Cas (ii). - Dans ce cas, on a pour tout $n$ assez grand

$$
\alpha_{n-1}=3 \alpha_{n}+\alpha_{n+1}
$$

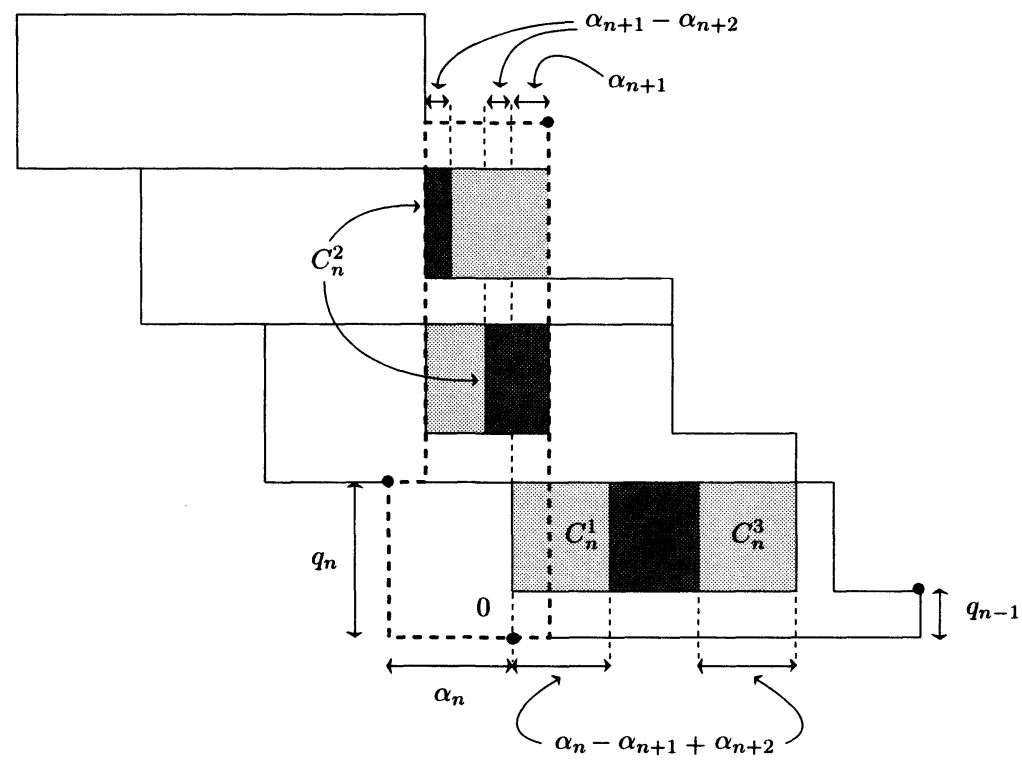

Figure 8

On divise alors $C_{n}$ suivant la figure 8 .

BULLETIN DE LA SOCIÉTÉ MATHÉMATIQUE DE FRANCE 
Si $\beta \in C_{n}^{1}$, l'égalité (3) donne

$$
\lambda\left(\left\{\phi^{\left(q_{n}\right)}=\varepsilon_{n}\right\}\right)=k_{n} \alpha_{n}+q_{n} x_{n} \leq q_{n}\left(2 \alpha_{n}-\alpha_{n+1}+\alpha_{n+2}\right) .
$$

Du lemme 2.2, il vient

$$
\begin{aligned}
\lim _{n \rightarrow \infty} q_{n}\left(2 \alpha_{n}-\alpha_{n+1}+\alpha_{n+2}\right) & =\frac{1}{\sqrt{13}}\left(2-\frac{(\sqrt{13}-3)}{2}+\frac{(\sqrt{13}-3)^{2}}{4}\right) \\
& =\frac{1}{\sqrt{13}}(9-2 \sqrt{13})<\frac{1}{2}
\end{aligned}
$$

d'où le résultat. Si $\beta \in C_{n}^{3}$, on obtient la même majoration par symétrie pour $\lambda\left(\left\{\phi^{\left(q_{n}\right)}=-\varepsilon_{n}\right\}\right)$. Si $\beta \in C_{n}^{2}$, on vérifie, à l'aide de l'induction sur $\mathcal{D}_{n}$ qu'on a $C_{n}^{2} \subset A_{n+1} \cup C_{n+1}^{1} \cup C_{n+1}^{3}$ et on est donc ramené aux cas précédents.

5.3.3. Cas (iii). - On décompose à nouveau $C_{n}$ suivant $A_{n+1}$ et $C_{n+1}$. Comme on a $a_{n+2}=1$, le lemme 4.2 règle les cas où $\beta \in C_{n} \cap C_{n+1}$ pour une infinité de $n \in \Lambda$. Sinon, on représente les parties $C_{n}^{1}, C_{n}^{2}$ et $C_{n}^{3}$ de $C_{n} \cap A_{n+1}$ selon la figure 9 .

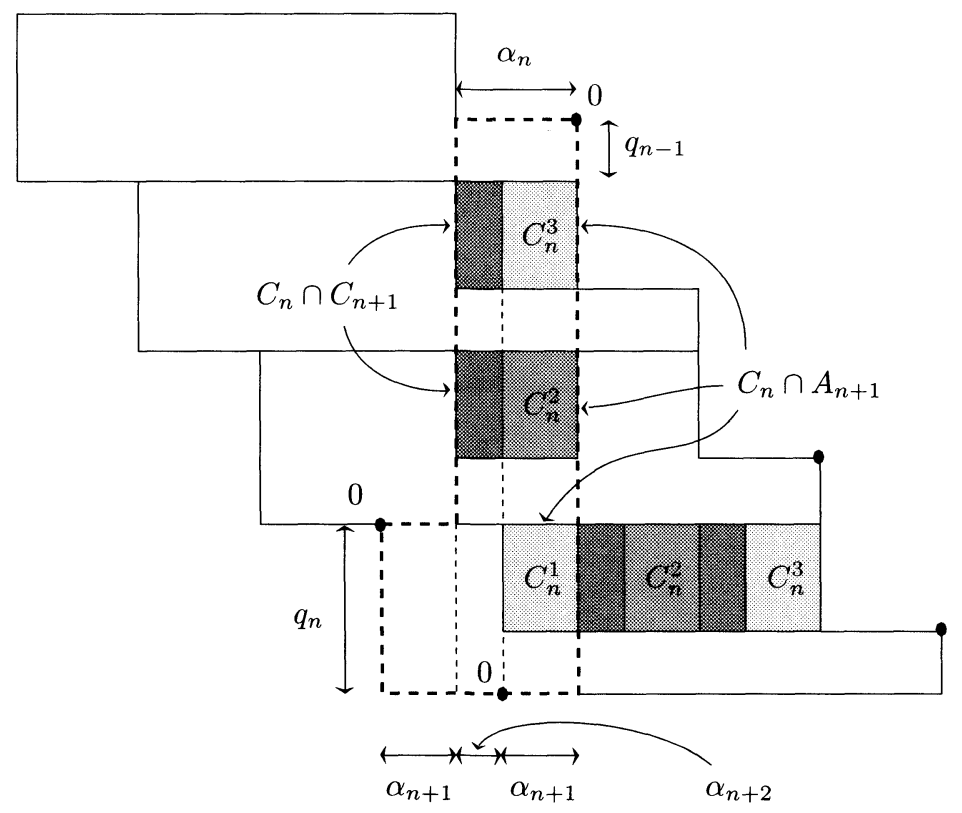

Figure 9

TOMe $127-1999-\mathrm{N}^{\circ} 1$ 
- Si $\beta \in C_{n}^{1}$, l'égalité $(3)$ pour $\phi^{\left(q_{n}\right)}$ donne

$$
\lambda\left(\left\{\phi^{\left(q_{n}\right)}=\varepsilon_{n}\right\}\right)=k_{n} \alpha_{n}+q_{n} x_{n} \leq\left(\alpha_{n}+\alpha_{n+1}\right) q_{n} .
$$

Comme $a_{n+1}=3$, on a

$$
\begin{aligned}
1 & =\alpha_{n} q_{n-1}+\alpha_{n-1} q_{n} \\
& =3 \alpha_{n} q_{n}+\alpha_{n+1} q_{n}+\alpha_{n} q_{n-1} \\
& \geq 2\left(\alpha_{n} q_{n}+\alpha_{n+1} q_{n}\right)+\alpha_{n} q_{n-1} .
\end{aligned}
$$

On en déduit alors $\varlimsup_{n \in \Lambda}\left(\alpha_{n}+\alpha_{n+1}\right) q_{n}<\frac{1}{2}$. S'il existe une infinité de $n \in \Lambda$ tels que $\beta \in C_{n}^{1}$, la proposition 1.1 est donc vraie.

- Si $\beta \in C_{n}^{3}$, on obtient par symétrie la même majoration que dans le cas précédent en remplaçant $\varepsilon_{n}$ par $-\varepsilon_{n}$.

- Si $\beta \in C_{n}^{2}$, comme $C_{n}^{2} \subset A_{n+1}$, on utilise l'égalité (4) à l'ordre $n+1$. Dans $\mathcal{D}_{n+1}, C_{n}^{2}=\left[0, \alpha_{n+1}\right] \times\left\{q_{n}+q_{n-1}+1, \ldots, 2 q_{n}\right\}$, et on obtient pour tout $\beta \in C_{n}^{2}$ :

$$
\begin{aligned}
\lambda\left(\phi^{\left(q_{n+1}\right)}\right. & \left.=\varepsilon_{n+1}\right) \\
& \leq 2 \alpha_{n+1} q_{n} \\
& \leq\left(\alpha_{n}+\alpha_{n+1}\right) q_{n} .
\end{aligned}
$$

Comme dans ce cas $\varlimsup_{n \in \Lambda}\left(\alpha_{n}+\alpha_{n+1}\right) q_{n}<\frac{1}{2}$, on en déduit le résultat voulu.

5.3.4. Cas (iv). - Dans ce cas, on redéfinit $\Lambda_{C}$ par l'un des ensembles infinis suivants :

(a) $\left\{n, \beta \in C_{n}, a_{n+1}=2\right.$ et $\left.a_{n+2}=1\right\}$.

(b) $\left\{n, \beta \in C_{n}, a_{n+1}=2\right.$ et $\left.a_{n+2} \neq 1\right\}$.

5.3.5. Cas (a). - On a $a_{n+1}=2$, et $a_{n+2}=1$. De même que dans le cas précédent, comme $a_{n+2}=1$, si $\beta \in C_{n} \cap C_{n+1}$ pour une infinité de $n \in \Lambda_{C}$, la proposition 1.1 résulte du lemme 4.2. Sinon, on a $\beta \in C_{n} \cap A_{n+1}$ pour tout $n \in \Lambda_{C}$ assez grand, et on peut donc utiliser l'égalité (4) à l'ordre $n+1$, soit

$$
\lambda\left(\phi^{\left(q_{n+1}\right)}=\varepsilon_{n+1}\right)=-x_{n+1}\left(q_{n+1}-k_{n+1}\right)+k_{n+1}\left(\alpha_{n+1}+x_{n+1}\right) .
$$

Ce cas est illustré par la figure 10, dans laquelle on a posé

$$
C_{n} \cap A_{n+1}=C_{n}^{1} \cup C_{n}^{2}
$$




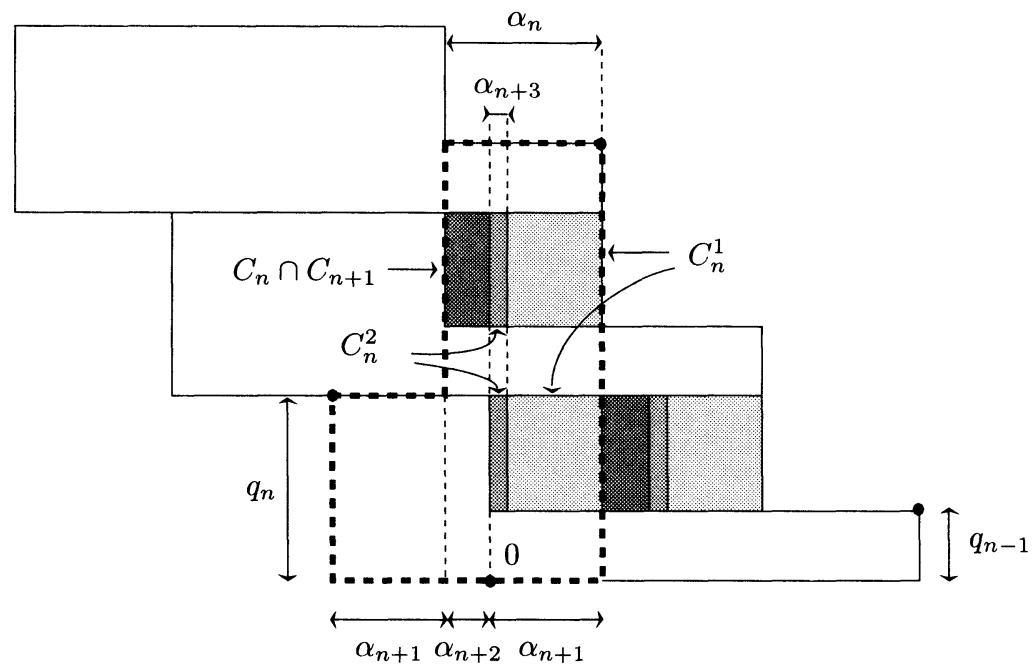

Figure 10

En utilisant l'induction entre $\mathcal{D}_{n}$ et $\mathcal{D}_{n+1}$, dans $\mathcal{D}_{n+1}$ on a $C_{n} \cap A_{n+1} \subset$ $\left[-\alpha_{n+1}, 0\right] \times\left\{q_{n-1}+1, \ldots, 2 q_{n}\right\}$ d'où $\lambda\left(\phi^{\left(q_{n+1}\right)}=\varepsilon_{n+1}\right) \leq 2 \alpha_{n+1} q_{n}$ pour tout $\beta \in C_{n} \cap A_{n+1}$.

Supposons que $a_{n+3}=1$ pour une infinité de $n \in \Lambda_{C}$, alors $2 \alpha_{n+2} \geq$ $\alpha_{n+1}$ et il vient

$$
\begin{aligned}
1 & =\alpha_{n} q_{n-1}+\alpha_{n-1} q_{n} \\
& =\alpha_{n} q_{n-1}+2 \alpha_{n} q_{n}+\alpha_{n+1} q_{n} \\
& =3 \alpha_{n+1} q_{n}+2 \alpha_{n+2} q_{n}+\alpha_{n} q_{n-1} \\
& \geq 4 \alpha_{n+1} q_{n}+\alpha_{n} q_{n-1} .
\end{aligned}
$$

On en déduit donc $\varlimsup_{n \in \Lambda_{C}} 2 \alpha_{n+1} q_{n}<\frac{1}{2}$, puis la proposition 1.1.

Sinon, pour tout $n \in \Lambda_{C}$ assez grand on a $a_{n+3} \neq 1$, et on pose

$$
C_{n} \cap A_{n+1}=C_{n}^{1} \cup C_{n}^{2}
$$

où $C_{n}^{1}$ et $C_{n}^{2}$ correspondent aux parties définies par la figure 10 et vérifient dans le repère d'ordre $n$

$$
\begin{aligned}
& C_{n}^{1} \subset\left[\alpha_{n+3}, \alpha_{n+1}\left[\times\left\{q_{n-1}+1, \ldots, 2 q_{n}\right\},\right.\right. \\
& C_{n}^{2} \subset\left[0, \alpha_{n+3}\right] \times\left\{q_{n-1}+1, \ldots, 2 q_{n}\right\} .
\end{aligned}
$$

TOME $127-1999-\mathrm{N}^{\circ} 1$ 
- Si $\beta \in C_{n}^{1}$ pour une infinité de $n \in \Lambda_{C}$, l'égalité (4) entraîne

$$
\lambda\left(\phi^{\left(q_{n+1}\right)}=\varepsilon_{n+1}\right) \leq 2 q_{n}\left(\alpha_{n+1}-\alpha_{n+3}\right) .
$$

Comme pour $n$ assez grand, $q_{n-1} \geq q_{n} / 4$ et $\alpha_{n+3} \geq \alpha_{n+2} / 4$, on a

$$
\begin{aligned}
1 & =\alpha_{n} q_{n+1}+\alpha_{n+1} q_{n} \\
& =\left(3 a_{n+3}+2\right) \alpha_{n+2} q_{n}+3 \alpha_{n+3} q_{n}+\alpha_{n} q_{n-1} \\
& \geq\left(3 a_{n+3}+2+3 / 4+\left(1+a_{n+3}\right) / 4\right) \alpha_{n+2} q_{n} \\
& \geq 4 a_{n+3} \alpha_{n+2} q_{n}+\alpha_{n+2} q_{n} / 4,
\end{aligned}
$$

d'où $\varlimsup_{n \in \Lambda_{C}} 2 q_{n}\left(\alpha_{n+1}-\alpha_{n+3}\right)=\varlimsup$

- Enfin, si $\beta \in C_{n}^{2}$, on a $\beta_{n+1}=\beta_{n+2}$, d'où $C_{n}^{2} \subset B_{n+2}$ et l'égalité (3) pour $\phi^{\left(q_{n+2}\right)}$ donne :

$$
\begin{aligned}
\lambda\left(\phi^{\left(q_{n+2}\right)}=\varepsilon_{n+2}\right) & =k_{n+1} \alpha_{n+2}-q_{n+2} x_{n+1} \\
& \leq \alpha_{n+2} q_{n+1}+\alpha_{n+3} q_{n+2} \\
& \leq \alpha_{n+2} q_{n+2}+\alpha_{n+3} q_{n+1}
\end{aligned}
$$

ce qui montre le résultat grâce au lemme $4.3\left(a_{n+3} \neq 1\right)$.

5.3.6 Cas (b). - On décompose $C_{n}$ sur $A_{n+1}$ et $C_{n+1}$. Dans ce cas comme $a_{n+2} \neq 1$, le lemme 4.3 démontre la proposition 1.1 lorsque $\beta \in C_{n} \cap A_{n+1}$ pour une infinité de $n \in \Lambda_{C}$. Sinon, on a $\beta \in C_{n} \cap C_{n+1}$ pour tout $n \in \Lambda$ assez grand. Posons dans ce cas

$$
h_{n}=q_{n-1}+q_{n}=q_{n+1}-q_{n} .
$$

Par un raisonnement analogue à celui du paragraphe 3.2, on obtient la répartition des valeurs de $\phi^{\left(h_{n}\right)}$ dans $\mathcal{D}_{n+1}$ suivant la figure 11 , lorsque $\beta$ varie dans $C_{n} \cap C_{n+1}$.

Soit $\delta_{n}$ le signe de $\phi^{\left(h_{n}\right)}$ dans la partie grise. Comme dans $\mathcal{D}_{n+1}$, on a

$$
C_{n} \cap C_{n+1}=\left[0, \alpha_{n}\right] \times\left\{q_{n}+q_{n-1}+1, \ldots, 2 q_{n}\right\},
$$

on obtient pour tout $\beta \in C_{n} \cap C_{n+1}$ :

$$
\begin{aligned}
\lambda\left(\phi^{\left(h_{n}\right)}=\delta_{n}\right) & =\left(\alpha_{n+1}+x_{n+1}\right)\left(k_{n+1}-q_{n}\right)+\left(\alpha_{n}-x_{n+1}\right)\left(q_{n+1}-k_{n+1}\right) \\
& \leq \alpha_{n} q_{n}+\alpha_{n+1} q_{n-1}
\end{aligned}
$$

et on conclut grâce au lemme 4.3.

BULlETIN DE LA SOCIÉTÉ MATHÉMATIQUE DE FRANCE 


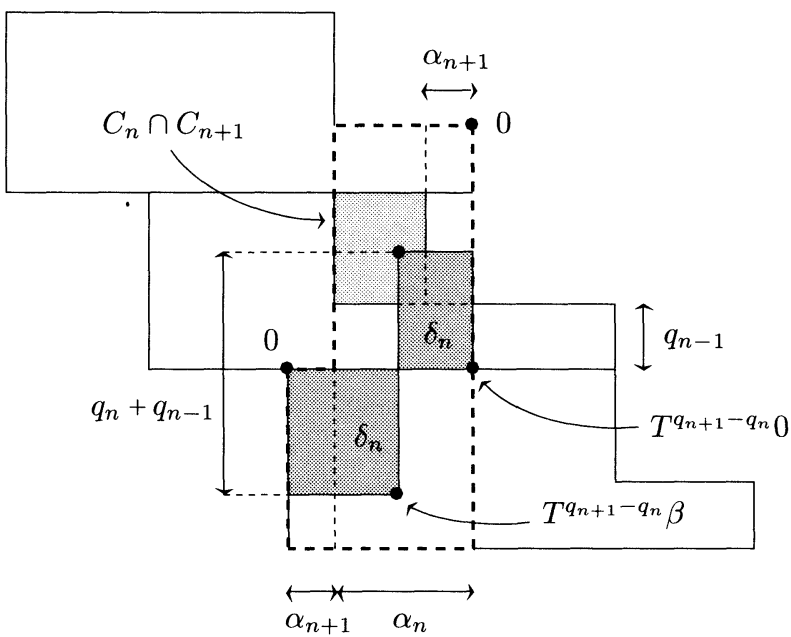

Figure 11

\section{BIBLIOGRAPHIE}

[1] Goodson (G.R.). - Approximations and spectral theory of finite skew products, J. London Math. Soc., t. 14, 1976, p. 249-259.

[2] Guenais (M.). - Une majoration de la multiplicité spectrale d'opérateurs associés à des cocycles réguliers, Israel J. Math., t. 105, 1998, p. 263-283.

[3] Helson (H.). - Cocycles on the circle, J. Operator Theory, t. 16, 1986, p. 189-199.

[4] Host (B.), Méla (J.F.), Parreau (F.). - Non singular transformations and spectral analysis of measures, Bull. Soc. Math. France, t. 119, 1991, p. 33-90.

[5] Host (B.), Parreau (F.). - The generalized purity law for ergodic measures : a simple proof, Colloq. Math., t. 60, 1990, p. 205-212.

TOME $127-1999-\mathrm{N}^{\circ} 1$ 
[6] Iwanik (A.), Lemańczyk (M.), Mauduit (C.). - Piecewise absolutely continuous cocycles over irrational rotations, à paraître dans J. London Math. Soc..

[7] KwiÁtKowski JR (J.), LemańCZYK (M.). - On the multiplicity function of ergodic group extensions, II, Studia Math., t. 116, 1995, p. 207-215.

[8] Katok (A.B.), Stepin (A.M.). - Approximations of ergodic dynamic systems by periodic transformations, Soviet. Math. Dokl., t. 7, 1966, p. $1638-1641$.

[9] Katok (A.B.), Stepin (A.M.). - Approximations in ergodic theory, Russian Math. Surveys, t. 22, 1967, p. 77-102.

[10] Khinchin (A.). - Continued Fractions. - University of Chicago Press, 1964.

[11] Merrill (K.D.). - Cohomology of step functions under irrational rotations, Israel J. Math., t. 52, 1985, p. 320-340.

[12] Oseledets (V.I.). - The spectrum of ergodic automorphisms, Soviet. Math. Dokl., t. 7, 1966, p. 776-779.

[13] Queffélec (M.). - Substitution Dynamical Systems, Spectral Analysis, in A. Dold and B. Eckmann, eds, Lecture Notes in Mathematics, vol. 1294, Springer-Verlag, 1987.

[14] RAuZy (G.). - Échanges d'intervalles et transformations induites, Acta Arithmetica, t. 34, 1979, p. 315-328.

[15] Riley (G.W.). - On spectral properties of skew products over irrational rotations., J. London Math. Soc., t. 17, 1978, p. 152-160.

[16] VeECH (W.A.). - Strict ergodicity in zero dimensional dynamical systems and the Kronecker-Weyl theorem modulo 2, Trans. Amer. Math. Soc., t. 140, 1969, p. 1-33. 